\title{
Factors affecting children's recognition memory for multidimensional stimuli
}

\author{
JOAN H. CANTOR and CHARLES C. SPIKER \\ University of Iowa, Iowa City, Iowa 52242
}

\begin{abstract}
The recognition memory of kindergarten and second-grade children was compared with respect to two different types of stimuli and under two different conditions of pretraining. For half of the children in each age group, the recognition-test stimuli were unitary stimuli of the standard type used in multidimensional (form, size, color) discrimination problems. For the other half, the stimuli were partitioned in that the values on the dimensions were spatially separated. Half of the children in each of these groups were trained to name the values on the stimulus compounds (size, color, shape). In the memory test, one of the eight stimulus compounds, which differed from each other with respect to one, two, or all three of the dimensions, was exposed to the child for $5 \mathrm{sec}$. It was then removed, and after 10 to $15 \mathrm{sec}$ the child was asked to find it among the complete set of eight compounds. The unitary stimuli were found to be more readily recognized than the partitioned stimuli by all children, the second-grade children recognized the partitioned stimuli, but not the unitary stimuli, better than did the kindergarten children, and the name training helped the kindergarten children in recognizing the partitioned stimuli. The memory data were discussed in terms of acquisition and retention differences.
\end{abstract}

Research on the hypothesis-testing capabilities of kindergarten and first-grade children has established two definite facts. First, the problem-solving capabilities of these children are significantly inferior to those of second-grade or older children, at least under normal conditions (e.g., Eimas, 1970; Gholson, Levine, \& Phillips, 1972, Kemler, 1978). Second, the hypothesistesting performance of the younger children can be markedly improved with training, either with supervised practice on discrimination problems or with training in the explicit testing of hypotheses (Cantor \& Spiker, 1978, 1979; Spiker \& Cantor, 1979). More recently, considerable attention has been turned to studying the more molecular aspects of the total discrimination task, such as the child's perception of and memory for the types of multidimensional stimuli widely used in such tasks.

One aspect of memory has been explored by supplying memory aids to the children in the discrimination task (Eimas, 1970; Kemler, 1978; Parrill-Burnstein, 1978; Spiker \& Cantor, in press). Considerable facilitation in performance occurred when conditions either

We wish to thank Christopher Reichl and Judith Vilmain for collecting and assisting in tabulating the data. Funds for the purchase of computer time were made available by the Graduate College of the University of Iowa. We are also deeply indebted to David L. Cronin, Superintendent of the Iowa City Community School District, for permission to test the children, to the principals of Coralville Central, Hoover, Horn, and Twain elementary schools, Stanley Bishop, Richard Hughes, Paul Davis, and Richard Hovet, and to their teachers and staffs, for their outstanding cooperation. Requests for reprints should be addressed to Joan H. Cantor, Department of Psychology, University of Iowa, Iowa City, Iowa 52242. forced or encouraged the children to make use of the memory aids, whereas no facilitation occurred following removal of the memory aids or under conditions in which they could be easily ignored by the children. These findings together with other data pertaining to memory in young children have led Kemler (1978) and others to a conclusion, with which we concur, that apparent deficiencies in the child's ability to remember the stimuli may actually be the child's failure to adopt a strategy of trying to remember the stimuli. In the present study, a direct test was made of the child's ability to remember the stimuli, eliminating the need for the child to adopt such a strategy.

The manner in which multidimensional stimuli are perceived by young children has also come under recent study. A number of investigators (e.g., Kemler \& Smith, 1978; Shepp \& Swartz, 1976; Smith \& Kemler, 1977), using speeded sorting tasks, have shown that stimuli that are perceived by older children and adults in terms of their dimensional structure tend to be perceived more as undifferentiated wholes by younger children. In a different context, we have also noted the apparent failure of many kindergarten children to analyze multidimensional stimuli into their dimensional components, as required in testing hypotheses in discrimination learning tasks (Spiker \& Cantor, in press). In an attempt to facilitate dimensional analysis for purposes of hypothesis testing (Cantor \& Spiker, 1978), we were therefore led to the use of stimuli with distributed or partitioned dimensions (Silleroy \& Johnson, 1973). Unlike the conventional unitary stimulus in which the dimensional values are combined in a single figure (e.g., a large blue circle centered on a card), the various dimensional 
values of partitioned stimuli are distributed spatially (e.g., a blue patch, a circular outline, and a large arrowhead all in different locations on a card).

The purpose of the present experiment was to assess the accuracy of the recognition memories of kindergarten and second-grade children for both unitary and partitioned types of three-dimensional stimuli, and to determine the effect of pretraining in labeling the dimensional values of such stimuli upon the accuracy of recognition memory. The criterion task was a delayed matchto-sample memory task in which the child was shown a stimulus compound (the target stimulus) for $5 \mathrm{sec}$; the compound was then removed, and after an additional $10-15 \mathrm{sec}$, the child was required to identify the target among a set of eight compounds displayed in a random order from trial to trial. Some children were given pretraining in which they were required to label the dimensional values of the target stimulus prior to each trial of a match-to-sample task; other children, although given the same match-to-sample pretraining, were not required to label the dimensional values. Pretraining the children to label the dimensional values prior to the memory task was designed to teach the child the value of making an explicit effort to remember the features of the target stimulus. Pretraining in labeling might also be expected to produce an encoding of the stimulus features that better resists decay over time, even for those children who are already trying to remember the features of the target stimulus. A factorial design was employed, with two types of training (labeling and control) and two types of stimuli (unitary and partitioned) within each of the two grade levels (kindergarten and second grade).

\section{METHOD}

\section{Subjects}

The subjects were 80 kindergarten children and 60 secondgrade children from the Iowa City Community School District, who participated on a voluntary basis after their parents had returned consent slips. The mean chronological age (CA) for the kindergarten children was 72.6 months, with a range from 65 to 82 months. For the second-graders, the mean CA was 97.7 months, with a range from 87 to 118 months. The data for one additional kindergarten child were excluded due to an experimenter error; the data for an additional four second-graders were excluded, two due to the children's lack of familiarity with the English language, one due to the child's very poor vision, and one due to the child's refusal to complete the experimental session. Children at both grade levels were randomly assigned in equal numbers to the groups representing the four combinations of stimulus type and training conditions.

\section{Stimulus Materials}

Three major types of stimuli were prepared, one type consisting of animal pictures for the preliminary training tasks, a second type consisting of the unitary multidimensional stimuli, and the third type consisting of the partitioned multidimensional stimuli. The animal pictures were eight colored drawings cut out from a children's book and attached to white cards, $6 \mathrm{~cm}$ square. There were two similar rabbits in different poses, two similar frogs in different poses, two mice flying similar airplanes, and two mice riding in similar boats.
The eight unitary stimuli consisted of all combinations of two colors (red and blue), two forms (square and circle), and two sizes (large and small). The dimensional values for each stimulus (e.g., large red square) were combined in a unitary figure cut out of colored cardboard and attached to the center of a white card, $6 \mathrm{~cm}$ square. The sides of the squares were $24 \mathrm{~mm}$ (large) and $14 \mathrm{~mm}$ (small), and the diameters of the circles were $25 \mathrm{~mm}$ (large) and $16 \mathrm{~mm}$ (small).

The same dimensional values constituting the unitary stimuli were used to prepare the partitioned stimuli. In this case, the three dimensional values for a given stimulus were spatially separated on the card, with a black line drawing of the appropriate form in the lower left corner, an irregular-shaped color patch in the lower right corner, and a vertical arrowhead representing size in the top center of the card. The forms had the same dimensions as the large stimuli previously described. The arrowhead was either $26 \mathrm{~mm}$ long (large) or $10 \mathrm{~mm}$ (small).

\section{Apparatus}

The apparatus consisted of a black wooden platform, $56 \mathrm{~cm}$ wide $\times 44 \mathrm{~cm}$ deep, which was bisected by a vertical partition $32 \mathrm{~cm}$ high. The apparatus was placed on a low table directly in front of the child, and the stimuli were presented on the front half of the platform, which was covered with white felt material. The stimulus materials not in use were placed out of the child's sight behind the partition.

\section{Procedure}

Preliminary match to sample. Each child was brought individually to a mobile research trailer located next to the school building and was seated in front of the apparatus. The preliminary training on matching to sample was the same for all groups. In order to simulate the stimulus settings of a standard simultaneous discrimination task, a pair of pictures was presented on each trial. In the first task, a pair of animal cards was presented on the platform, and a large white card $(35.5 \mathrm{~cm}$ wide $\mathrm{x} 20 \mathrm{~cm}$ high) containing the full set of animal pictures was displayed by standing it on a ledge centered in the vertical partition. The experimenter pointed to one of the two pictures (the target stimulus) and asked the child to point to the one exactly like it on the large card. The experimenter immediately removed the second card; thus, the child matched only one target stimulus on each trial. Eight such trials were presented, with each of the eight pictures serving as the target stimulus once. When occasional errors occurred, mainly when a stimulus similar to the target was selected, the child was required to find the correct match.

Match to sample. The appropriate unitary or partitioned multidimensional stimuli were introduced in the subsequent match-to-sample task, using the same general procedures. The full set of eight cards was displayed on a large white card, and the children were told that now there were different colors, shapes, and sizes. The experimenter named each of the six dimensional values and identified each by pointing.

At this point, special training was begun for the labeling groups. Basically, the training required the child to name in succession the values of each of the three dimensions represented on the card. Although children of these ages find it easy to name the different values of a single dimension on each of several cards (e.g., "red," "blue," "green," etc.), a task that requires them to name the values on all the dimensions of each of several successive stimuli proves to be considerably more difficult (e.g., "big red square," "little blue square," "big blue circle," etc.). The experimenter began the training in labeling by pointing to and naming each card in turn; for example, "This card has a little blue circle." For the partitioned stimuli, the experimenter pointed successively to the spatially separated features. The child was then asked to repeat the naming for each stimulus, and any errors were corrected. The child was encouraged by example to label the dimensional values in the order size, color, shape, although other orders were accepted 
and were not considered errors. Labeling practice continued until the child named all eight stimuli correctly without hesitation. The match-to-sample task proceeded in the manner described above. The child was required to name the dimensional values on the target stimulus before looking for the match. Following the child's response, the experimenter held the target card under the card selected and provided feedback. For correct responses, the experimenter said, "Very good. They are both (size, color, form)." For incorrect responses, the feedback was, "No. this is a _. The one you picked is a

Find the right one." Eight trials were given, with each stimulus being matched once.

In the control groups, the same procedures were followed except that no labeling practice was given, and the stimulus was not named prior to the matching response. The feedback for correct responses was, "Very good. They are the same." For incorrect responses, it was, "No, they aren't the same. Find the right one."

Delayed match to sample. Following the match-to-sample task, the large white display card was removed. The experimenter then said, "From now on, let's see if you can remember the one I point to. I'll give you some time so you can look at it carefully and try to remember it." On each trial, the experimenter pointed to the target stimulus, waited $5 \mathrm{sec}$, and covered it with a card. The experimenter then shuffled a full set of stimulus cards, laid them out in a straight line on the platform, and asked the child to point to the matching stimulus. The delay period during which the child had to remember the covered stimulus varied between 10 and $15 \mathrm{sec}$. No labeling was used in any group, and no feedback was given. The performance criterion in the memory task was six consecutive correct responses, with a minimum of 8 trials and a maximum of 16 trials.

\section{RESULTS}

\section{Matching To Sample}

There was an unequivocal difference in the matchto-sample performance of children who received partitioned stimuli as compared with those who received unitary stimuli. Whereas only 1 child out of $70(1 \%)$ made at least one error in matching the unitary stimuli, 36 children $(51 \%)$ did so with the partitioned stimuli, a difference that is significant at less than the .001 level $\left[\chi^{2}(1)=42.5\right]$. The mean number of errors made in the eight match-to-sample trials with partitioned stimuli was .89 . The mean number of errors for children who received name training (.54) was significantly lower than that for the children who did not receive such training (1.23) $[F(1,67)=21.63, p<.001]$. The difference between kindergarten and second-grade children, however, was not significant $[F(1,67)=1.96, p>.10]$. For the partitioned stimuli, over two-thirds of the errors were made during the first half of the trials.

\section{Delayed Matching to Sample}

The proportion of correct matches was determined for each child and entered into an analysis of variance, with type of stimulus, labeling pretraining, and grade level as between-subjects factors. The means for the various subgroups are shown in Table 1 . The ANOVA revealed that every main effect and interaction was significant at less than the .05 level, including the triple interaction involving stimulus type, grade, and pretraining. Since the second-graders were significantly superior
Table 1

Mean Proportion of Correct Matches for the Kindergarten and Second-Grade Children for the Partitioned and Unitary Stimuli in the Control (C) and Labeling ( $L$ ) Conditions

\begin{tabular}{lccccccc}
\hline \multirow{2}{*}{$\begin{array}{c}\text { Stimulus } \\
\text { Type }\end{array}$} & \multicolumn{3}{c}{ Kindergarten } & & \multicolumn{3}{c}{ Second Grade } \\
\cline { 2 - 4 } \cline { 6 - 8 } & $\mathrm{C}$ & $\mathrm{L}$ & $\mathrm{C}+\mathrm{L}$ & & $\mathrm{C}$ & $\mathrm{L}$ & $\mathrm{C}+\mathrm{L}$ \\
\hline Partitioned & .527 & .786 & .657 & & .869 & .893 & .881 \\
Unitary & .913 & .909 & .911 & & .931 & .940 & .935 \\
Combined & .720 & .848 & .784 & & .900 & .917 & .908 \\
\hline
\end{tabular}

to the kindergarten children, and since the triple interaction was significant, the proportion of correct matches was analyzed separately for the two grade levels.

For the kindergarten children, the effect of labeling, type of stimulus, and their interaction were all significant at less than the .01 level, with $F$ ratios of $10.26,40.72$, and 10.86 , respectively, with $\mathrm{df}=1$ and 76 . It can be seen from Table 1 that the children given the labeling pretraining were superior to the control subjects and that the children recognized the unitary stimuli more accurately than the partitioned stimuli. The interaction of pretraining and stimulus type reflects the fact that the labeling enhanced recognition of the partitioned stimuli but had no discernible effect on recognition of the unitary stimuli. For the second-grade children, the only significant effect was for stimulus type $[F(1,56)=5.72, p=.02]$. Table 1 indicates that the effect of pretraining was minor for the second-graders and that the training did not interact with type of stimulus.

\section{DISCUSSION}

The results from the match-to-sample phase indicate clearly that the children of both age groups were able to identify the unitary-stimulus targets with greater accuracy than could their counterparts who were given the partitioned-stimulus targets. Since both the target stimulus and the selection set were in full view, the memory requirements in the match-to-sample task would seem to be minimal. Thus, it seems likely that the difference in performance reflects a difference in perceptual processing.

The partitioned stimuli differed from the unitary stimuli in several respects, and it is impossible to isolate any one aspect as that which produced performance differences. For example, the relevant features of the partitioned stimuli were more widely distributed in space, requiring that a greater area be scanned in order to obtain all the relevant information. Moreover, the number of irrelevant features was considerably greater for the partitioned stimuli than for the unitary stimuli. Either of these factors would probably require a more complex visual orientation for the partitioned stimuli than would be required for the unitary stimuli. One consequence of such circumstances might be that more time was needed in making a match when the stimuli were partitioned, although the present study provides no information on this hypothesis. Another expected consequence is that children working with partitioned stimuli, relative to those given the unitary stimuli, would make erroneous identifications more frequently as a result of having failed to obtain all the relevant information, either from the target or from the stimulus chosen. The superior matching performance with partitioned stimuli that was observed for those children who 
received name training might then be attributed to the development of a more reliable orienting routine.

Interpretation of the results from the recognition memory phase must be made with an apparent ceiling effect in mind. Thus, for the unitary stimuli, there were no differences either in grade level or in pretraining. It is quite possible, however, that more complex unitary stimuli-perhaps with different dimensions or a larger number of dimensions-might reveal age differences and/or pretraining effects. Although both age groups showed poorer recognition of the partitioned stimuli, the kindergarten children were markedly inferior to the second-graders, particularly in the control group, in which only approximately half of the choices were correct. Pretraining the kindergarten children to label the dimensional values of the target stimuli produced marked facilitation in recognition of the partitioned stimuli. Although naming the partitioned stimuli did not significantly improve performance of the second-graders, it is again possible that a more complex task might reveal naming effects.

The use of a recognition memory task makes it safe to assume that differences in retrieval are not responsible for the observed differences in remembering partitioned and unitary stimuli. The differences, however, may still be attributed to differences in the accuracy of extracting the relevant information from the two types of stimuli (acquisition), to differences in the strength or vigor of the memory traces at testing time (retention), or to both. Fairly cogent arguments can be found for both alternatives, although the present data do not provide a means for choosing between them. The hypothesis that the observed differences arose from differences in acquisition derives its most compelling support from the results of the match-to-sample task-the partitioned stimuli were more difficult to identify even when all stimuli were in full view. The argument that performance differences arose from differences in retention is most strongly supported by the fact that pretraining in labeling the partitioned stimuli, which might be expected to optimize acquisition, did not result, even for second-graders, in a performance level equal to that achieved by children who received the unitary stimuli.

Either of the two hypotheses has a ready explanation for the effect of the label pretraining. The acquisition account suggests that requiring the labeling of the partitioned stimuli forces the child to develop a reliable, systematic scanning routine. Labeling the unitary stimuli has no effect because it is easy to extract the relevant information from them. The retention account suggests that labeling the partitioned stimuli produces a verbal code that resists the temporal decay better than does the visual trace for these stimuli. Labeling the unitary stimuli has no effect, probably because the visual trace is quite adequate for the relatively short delay period or, possibly, because a verbal code is normally elicited by these stimuli.

Finally, the present results add new information to results from other laboratories concerning the conditions under which children perceive stimulus dimensions in either a separable or integral manner (e.g., Kemler \& Smith, 1978, 1979; Shepp \& Swartz, 1976; Smith \& Kemler, 1977). Our results suggest that the unitary stimuli are perceived, for purposes of recognition memory, in a more integral unanalyzed fashion and are probably carried primarily in a visual code. The nature of the partitioned stimuli, on the other hand, appears to necessitate perception in a more separable fashion and to require verbal encoding for purposes of recognition memory. The relatively poor performance of the kindergarten children with the partitioned stimuli is thus consistent with earlier findings that younger children tend to perceive stimuli less separably than do older children and adults. In addition, the marked facilitation in kindergarten performance with practice in labeling is also consistent with the conclusion (Kemler \& Smith, 1978) that these children can and do deal effectively with the dimensional structure of stimuli under certain conditions.

\section{REFERENCES}

Cantor, J. H., \& Spiker, C. C. The problem-solving strategies of kindergarten and first-grade children during discrimination learning. Journal of Experimental Child Psychology, 1978, 26, 341-358.

Cantor, J. H., \& Spiker, C. C. The effects of introtacts on hypothesis testing in kindergarten and first-grade children. Child Development, 1979, 50, 1110-1120.

Eimas, P. D. Effects of memory aids on hypothesis behavior and focusing in young children and adults. Journal of Experimental Child Psychology, 1970, 10, 319-326.

Gholson, B., Levine, M., \& Phillips, S. Hypotheses, strategies, and stereotypes in discrimination learning. Journal of Experimental Child Psychology, 1972, 13, 423-446.

Kemler, D. G. Patterns of hypothesis testing in children's discriminative learning: A study of the development of problemsolving strategies. Developmental Psychology, 1978, 14, 653-673.

KemLer, D. G., \& Sмith, L. B. Is there a developmental trend from integrality to separability in perception? Journal of Experimental Child Psychology, 1978, 26, 498-507.

Kemler, D. G., \& Smith, L. B. Accessing similarity and dimensional relations: Effects of integrality and separability on the discovery of complex concepts. Journal of Experimental Psychology: General, 1979, 108, 133-150.

Parrill-Burnstein, M. Teaching kindergarten children to solve problems: An information-processing approach. Child Development, 1978, 49, 700-706.

Shepp, B. E., \& Swartz, K. B. Selective attention and the processing of integral and nonintegral dimensions. Journal of Experimental Child Psychology, 1976, 22, 73-85.

Silleroy, R. S., \& Johnson, P. J. The effects of perceptual pretraining on concept identification and preference. Journal of Experimental Child Psychology, 1973, 15, 462-472.

Smith, L. B., \& Kemler, D. G. Developmental trends in free classification: Evidence for a new conceptualization of perceptual development. Journal of Experimental Child Psychology, 1977, 24, 279-298.

Spiker, C. C., \& Cantor, J. H. Factors affecting hypothesis testing in kindergarten children. Journal of Experimental Child Psychology, 1979, 28, 230-248.

Spiker, C. C., \& Cantor, J. H. Cognitive strategies in the discrimination learning of young children. In D. K. Routh (Ed.), Learning, speech, and the complex effects of punishment. New York: Plenum, in press.

(Received for publication August 20, 1980.) 